\title{
Adenomyosis is an independent risk factor for complications in deep endometriosis laparoscopic surgery
}

\section{Meritxell Gracia}

Hospital Clínic de Barcelona

Cristian de Guirior

Hospital Clínic de Barcelona

Marta Valdés-Bango

Hospital Clínic de Barcelona

Mariona Rius

Hospital Clínic de Barcelona

Cristina Ros

Hospital Clínic de Barcelona

Isabel Matas

Hospital Clínic de Barcelona

Marta Tortajada

Hospital Clínic de Barcelona

María Ángeles Martínez-Zamora

Hospital Clínic de Barcelona

Lara Quintas

Hospital Clínic de Barcelona

Francisco Carmona ( $\sim$ fcarmona@clinic.cat)

Hospital Clínic de Barcelona

\section{Research Article}

Keywords: Clavien-Dindo, laparoscopy, complications, outcome, surgery, adenomyosis

Posted Date: September 15th, 2021

DOI: https://doi.org/10.21203/rs.3.rs-893239/v1

License: (c) (1) This work is licensed under a Creative Commons Attribution 4.0 International License.

Read Full License 


\section{Abstract \\ Background}

Deep endometriosis (DE) occurs in 15-30\% of patients with endometriosis and is associated with concomitant adenomyosis in around $49 \%$ of cases. There are no data about the effect of the presence of adenomyosis in terms of surgical outcomes and complications. Thus, the aim of the present study was to evaluate the impact of adenomyosis on surgical complications in women with deep endometriosis undergoing laparoscopic surgery.

\section{Methods}

A retrospective cohort study including women referred to the endometriosis unit of a referral teaching hospital. Two expert sonographers preoperatively diagnosed DE and adenomyosis. DE was defined according to the criteria of the International Deep Endometriosis Analysis group. Adenomyosis was considered when 3 or more ultrasound criteria of the Morphological Uterus Sonographic Assessment group were present. Demographical variables, current medical treatment, symptoms, DE location, surgical time, hospital stay and difference in pre and post hemoglobin levels were collected. The Clavien-Dindo classification was used to assess surgical complications, and multivariate analysis was performed to compare patients with and without adenomyosis.

\section{Results}

157 DE patients were included into the study; 77 (49.05\%) had adenomyosis according to transvaginal ultrasound (TVS) and were classified in the A group, and 80 (50.95\%) had no adenomyosis and were classified in the noA group. Adenomyosis was associated with a higher rate of surgical complications: $33.76 \%$ (A group) vs. $12.5 \%$ (noA group) $(p<0.001$ ). Multivariate analysis showed a 4.56 -fold increased risk of presenting complications in women with adenomyosis (Cl: 1.9-11.3; $p=0.001)$ independently of undergoing hysterectomy. There was a statistically significant association between the number of criteria of adenomyosis present in each patient and the proportion of patients presenting surgical complications $(p<0.001)$.

\section{Conclusions}

Adenomyosis increases the risk of presenting complications in DE surgery after controlling for demographic, clinical and surgical factors and should be considered an independent preoperative risk factor of surgical complications.

\section{Introduction}


For years endometriosis and adenomyosis were related, but they are now mainly considered as separate entities [1, 2]. Deep endometriosis (DE) occurs in 15-30\% of patients with endometriosis and is associated with concomitant adenomyosis in around $49 \%$ of cases $[3,4]$.

Advances in pelvic ultrasound provide a high accuracy in the diagnosis of the different forms of endometriosis [5, 6] and adenomyosis [7-9] and allow topographical planning prior to surgery $[10,11]$.

Hormone therapy is the first line treatment for DE and adenomyosis in patients not seeking pregnancy. When severe pain persists following medical treatment, or in cases of intestinal or ureteral obstruction, conservative or radical surgical approaches may be necessary depending on the need for fertility preservation $[12,13]$. Surgery has been widely demonstrated to improve endometriosis-related symptoms [14]. However, resection of endometriotic lesions is often challenging. Most of DE interventions are highly complex and are associated with a significant risk of complications that must be preoperatively taken into account [15]. Moreover, there is no a reliable preoperative marker to determine the severity of endometriosis for extrapolation to surgical difficulty [16]. To the best of our knowledge, there are no data about the effect of the presence of adenomyosis in terms of surgical outcomes and complications.

Thus, the aim of the present study was to assess the impact of adenomyosis on the presentation of surgical complications in patients with DE undergoing laparoscopic surgery.

\section{Materials And Methods}

A retrospective cohort study including women referred to the Endometriosis Unit of the Hospital Clinic of Barcelona, who underwent DE surgery from July 2018 to December 2019 was designed.

The study was approved by the Ethical Committee of the Hospital Clinic (Reg: HCB/2019/1152), all research was performed in accordance with relevant guidelines and regulations and informed consent was obtained from all patients.

A preoperative diagnosis of DE and adenomyosis was made by two experts sonographers within 6 months prior to surgery. DE was described according to the fourth step method suggested by the International Deep Endometriosis Analysis (IDEA) group [6] with a 2-dimensional and 3-dimensional transvaginal sonography (TVS) using an endovaginal probe (type RIC5-9, Voluson V730 Expert; GE Healthcare, Milwaukee, WI) with previous bowel preparation [5]. The location and extent of DE was described within the pelvis: rectovaginal septum, torus uterinus, uterosacral ligaments, vaginal fornix, bladder, ureteral and bowel involvement. Adenomyosis ultrasound features according to the criteria of the Morphological Uterus Sonographic Assessment (MUSA) group [7], were: asymmetrical thickening, cysts, hyperechoic islands, fan-shaped shadowing, echogenic subendometrial lines and buds, translesional vascularity, irregular junctional zone and interrupted junctional zone. Adenomyosis was diagnosed when at least 3 of the above-mentioned ultrasound features were present according to our hospital protocol. 
DE surgery was always performed by the same team of skilled endometriosis surgeons and a colorectal surgeon or urologist when needed. In order to standardize all the surgical procedures, they were classified into: adnexal (including salpingectomy, ovarian cystectomy or $\mathrm{CO} 2$ laser vaporization and adnexectomy), pelvic (vagina, uterosacral ligaments, torus uterinus and rectovaginal septum), bowel (shaving, discoid or segmental resection), urinary (ureterolysis, bladder nodule excision, nephrectomy) and hysterectomy. Some patients underwent more than one of these procedures. In addition, endometriosis was staged according to the revised-American Society of Reproductive Medicine (r-ASRM) classification score [17].

The following demographic data were collected: age, body mass index (BMI), previous endometriosis surgery, infertility, parity, preoperative hormonal treatment and surgical indication. The endometriosisrelated symptoms considered were: dysmenorrhea, dyspareunia, dyschezia, dysuria and non-cyclic pelvic pain, using a numerical rating scale (NRS) in which 0 was no pain and 10 unbearable pain. Abnormal uterine bleeding (AUB) was also registered.

Patients were divided into two groups according to the preoperative TVS features: with adenomyosis (A) or without adenomyosis (noA). The main goal of the study was to compare postoperative complications in the two groups according to the Clavien-Dindo (CD) classification [18]. Differences in demographic characteristics, medical treatment, symptoms, DE location, types of DE surgery, surgical time (minutes), hospital stay (days) and pre and post hemoglobin levels ( $\mathrm{gr} / \mathrm{dl})$ were also assessed.

\section{Statistical analysis}

Statistical analysis was performed using SPSS v 21.0 software (IBM, Armonk, NY, USA). Patient characteristics were described using frequency tables for nominal variables and measures of central tendency and dispersion for continuous variables.

To compare outcomes between the two groups, the Chi-squared or Fisher exact tests was used for categorical data, and continuous variables were compared using independent t-tests or the Mann-Whitney test as appropriate. Bivariate logistic regression analysis was used to determine the factors related to the incidence of surgical complications. All bivariate statistical tests were performed at a significance level of $p<0.05$ (two-sided). Independent variables that were related to complications in the bivariate analysis with a $p<0.15$ were candidates for inclusion in the multivariate logistic regression model. This model explained the probability of the presentation of surgical complications with respect to non-presentation of complications as a function of the variables included. For the multivariate analysis, up to $p<0.10$ was illustrated.

Furthermore, we also conducted a subanalysis of the complications between groups considering patients without hysterectomy.

\section{Results}


A total of 157 patients undergoing DE surgery were included during the study period. According to TVS, 77 (49.05\%) patients had 3 or more adenomyosis criteria and were classified in the A group and 80 (50.95\%) patients had less than 3 adenomyosis criteria and were classified in the noA group.

No differences were observed between groups regarding age, BMI and parity (Table 1). A total of $52.86 \%$ of women had one or more previous endometriosis surgeries, with no significant differences between groups. Among the 157 patients studied, 130 (82.81\%) received preoperative continuous hormonal medical treatment, mainly with oral combined contraceptives, with no significant differences between groups. 
Table 1

Demographic characteristics and endometriosis-related symptoms

\begin{tabular}{|c|c|c|c|}
\hline & $\begin{array}{l}\text { ADENOMYOSIS } \\
\text { (A) } \mathrm{N}=77\end{array}$ & $\begin{array}{l}\text { NO ADENOMYOSIS (noA) N= } \\
80\end{array}$ & $\begin{array}{l}\mathrm{P} \\
\text { Value }\end{array}$ \\
\hline Age, years $($ mean $\pm S D)$ & $38.22 \pm 6.63$ & $37.64 \pm 5.85$ & NS \\
\hline $\mathrm{BMI} \mathrm{kg} / \mathrm{m}^{2}($ mean $\pm \mathrm{SD})$ & $24.45 \pm 4.98$ & $23.90 \pm 5.41$ & NS \\
\hline Parity (mean \pm SD) & $0.43 \pm 0.81$ & $0.41 \pm 0.83$ & NS \\
\hline Infertility & 36 (46.75) & $28(35)$ & NS \\
\hline Hormonal treatment n (\%): & 14 (18.18) & $13(16.25)$ & NS \\
\hline None & $32(41.55)$ & $36(45)$ & \\
\hline Combined contraceptives & $13(16.88)$ & $13(16.25)$ & \\
\hline Progestins & $5(6.49)$ & $3(3.75)$ & \\
\hline LNG-IUD & $13(16.88)$ & $15(18.75)$ & \\
\hline \multicolumn{4}{|l|}{$\mathrm{aGnRH}$} \\
\hline DE location n (\%): & $49(63)$ & 27 (33.75) & \multirow[t]{8}{*}{ NS } \\
\hline Torus uterinus & 16 (20.77) & $10(12.5)$ & \\
\hline Vaginal fornix & $44(57.14)$ & $32(40)$ & \\
\hline Uterosacral ligaments & $5(6.49)$ & $6(7.5)$ & \\
\hline Rectovaginal septum & $14(18.18)$ & $12(15)$ & \\
\hline Ureteral + bladder & 35 (45.45) & $32(40)$ & \\
\hline Bowel & $60(77.9)$ & $52(65)$ & \\
\hline \multicolumn{3}{|l|}{ Ovarian endometrioma } & \\
\hline \multirow{2}{*}{$\begin{array}{l}\text { Previous endometriosis surgery } n \\
\text { (\%): }\end{array}$} & $33(42.85)$ & $41(51.25)$ & \multirow[t]{4}{*}{ NS } \\
\hline & $34(44.15)$ & $33(41.25)$ & \\
\hline 1 procedure & $10(12.98)$ & $6(7.5)$ & \\
\hline$\geq 2$ procedures & & & \\
\hline \multicolumn{4}{|c|}{$\begin{array}{l}\text { Data are given as } n \text { or \%. Symptoms are expressed with Numeric Rating Scale (NRS). LNG-IUD: } \\
\text { Levonorgestrel Intra Uterine Device; aGnRH: analog-Gonadotropin-releasing Hormone; SD: standard } \\
\text { deviation; n: number; \%: percentage; AUB: Abnormal Uterine Bleeding; NS: non-significant; BMI: body } \\
\text { mass index }\end{array}$} \\
\hline \multicolumn{4}{|c|}{$\begin{array}{l}\text { *Dysmenorrhea NRS was reported in patients with regular monthly periods: } 14 \text { patients in A group } \\
(18.18 \%) \text { and } 13 \text { in noA Group }(16.25 \%)\end{array}$} \\
\hline
\end{tabular}




\begin{tabular}{|c|c|c|c|}
\hline & $\begin{array}{l}\text { ADENOMYOSIS } \\
\text { (A) } \mathrm{N}=77\end{array}$ & $\begin{array}{l}\text { NO ADENOMYOSIS (noA) N= } \\
80\end{array}$ & $\begin{array}{l}\mathrm{P} \\
\text { Value }\end{array}$ \\
\hline AUB n (\%): & $48(62.33)$ & $51(63.75)$ & NS \\
\hline No & $29(37.66)$ & $29(36.25)$ & \\
\hline \multicolumn{4}{|l|}{ Yes } \\
\hline Dysmenorrhea* $($ mean \pm SD) & $7.20 \pm 3.13$ & $6.85 \pm 3.03$ & NS \\
\hline Dyspareunia (mean \pm SD) & $4.72 \pm 4.05$ & $4.61 \pm 3.84$ & NS \\
\hline \multicolumn{4}{|l|}{$($ mean $\pm S D)$} \\
\hline Non-cyclic pelvic pain (mean \pm SD) & $4.69 \pm 3.50$ & $4.09 \pm 3.49$ & NS \\
\hline Dysuria (mean \pm SD) & $1.22 \pm 2.81$ & $1.02 \pm 2.41$ & NS \\
\hline \multicolumn{4}{|c|}{$\begin{array}{l}\text { Data are given as } n \text { or \%. Symptoms are expressed with Numeric Rating Scale (NRS). LNG-IUD: } \\
\text { Levonorgestrel Intra Uterine Device; aGnRH: analog-Gonadotropin-releasing Hormone; SD: standard } \\
\text { deviation; n: number; \%: percentage; AUB: Abnormal Uterine Bleeding; NS: non-significant; BMI: body } \\
\text { mass index }\end{array}$} \\
\hline
\end{tabular}

Table 1 shows the endometriosis-related symptoms of both study groups. The mean of all NRS scores and the presence of AUB, although not significant, were higher (except for dyschezia) in the group of patients with adenomyosis (group A). The main indication for surgery was pain in both groups (96\%) despite receiving medical treatment, with $46.75 \%$ (group A) and $35 \%$ (group no A) having associated infertility ( $p$ 0.495).

The different interventions performed in the two groups are shown in Table 2. All the procedures were performed by laparoscopy with 56 hysterectomies being registered: 38 in the A group and 18 in the noA group ( $p$ 0.002). DE was confirmed histologically in all cases as well as the presence of adenomyosis when hysterectomy or uterus-sparing adenomyosis surgery was performed. 
Table 2

Surgical procedures and r-ASRM classification

ADENOMYOSIS NO ADENOMYOSIS (nOA) $\mathbf{N}=\mathbf{8 0}$

P Value

(A) $\mathrm{N}=77$

Type of surgery $n(\%)$ :

$72(93.5)$

67 (83.75)

NS

Adnexal surgery *:

$55(71.42)$

$45(56.25)$

NS

Pelvic DE ${ }^{+}$:

$22(28.57)$

19 (23.75)

NS

Bowel Surgery:

13 (16.88)

13 (16.25)

NS

- Shaving

$6(7.5)$

NS

- Segmental resection

$9(11.68)$

$6(7.5)$

NS

Urinary Surgery:

38 (49.35)

$18(22.5)$

.002

- Bladder nodule excision

- Ureterolysis/reimplantation

Hysterectomy: 56/157 (35.66)

r-ASRM score n (\%):

3 (3.89)

$1(1.25)$

NS

I

9 (11.25)

II

$12(15.58)$

19 (23.75)

III

58 (75.32)

51 (63.75)

IV

* Adnexal surgery: unilateral or bilateral including ovarian endometriomas, salpingectomy, adnexectomy. ${ }^{+}$Pelvic DE: including vagina, uterosacral ligaments, torus uterinus.

Data are given as n or \%. r-ASRM score: revised-American Society of Reproductive Medicine; SD: standard deviation; n: number; \%: percentage; NS: non-significant

The presence of adenomyosis showed significant differences in the CD complications rates: A group $33.76 \%$, noA group 12.5\% ( $\mathrm{p} 0.001$ ), mean surgical time: A group $231 \pm 101$ minutes, noA group $181.08 \pm$ 91.61 minutes ( $p$ 0.011) and difference in pre-post hemoglobin levels: A group $2.17 \pm 1.89 \mathrm{gr} / \mathrm{dl}$, and noA group $2.05 \pm 1.22 \mathrm{gr} / \mathrm{dl}(\mathrm{p} 0,049)$. There were no significant differences in length of hospital stay: A group $3.32 \pm 3.7$ days, noA group $2.75 \pm 1.85$ days (p 0.09). Most of the complications were minor $(88.8 \% \mathrm{CD}$ I and II) (Table 3). Four patients in the A group, presented major complications (CD III): one rectovaginal fistula, two anastomotic leakages and one bowel obstruction. All patients were recovered at 3-6 months of post-operative follow-up. 
Table 3

Surgical data and complications

\begin{tabular}{|llll|}
\hline & $\begin{array}{l}\text { ADENOMYOSIS (A) } \\
\text { N }=77\end{array}$ & $\begin{array}{l}\text { NO ADENOMYOSIS } \\
\text { (nOA) N=80 }\end{array}$ & $\begin{array}{c}\text { P } \\
\text { Value }\end{array}$ \\
\hline Surgical time minutes (mean \pm SD) & $231 \pm 101$ & $181.08 \pm 91.61$ & .011 \\
\hline $\begin{array}{l}\text { Difference pre-post Hemoglobin levels } \\
\text { g/dl (mean } \pm \text { SD) }\end{array}$ & $2.17 \pm 1.89$ & $2.05 \pm 1.22$ & .049 \\
\hline Hospital Stay days (mean \pm SD) & $3.32 \pm 3.7$ & $2.75 \pm 1.85$ & .09 \\
\hline CD Complications & $26(33.76)$ & $10(12.5)$ & \\
n (\%) & $12(15.58)$ & $4(5)$ & \\
I & $10(12.98)$ & $6(7.5)$ & \\
II & $4(5.19)$ & $0(0)$ & \\
III & $0(0)$ & $0(0)$ & \\
IV & & & \\
\hline Data are given as $n$ or \%. SD: standard deviation; CD: Clavien-Dindo; $\mathrm{n}$ : number; \%:percentage & \\
\hline
\end{tabular}

Multiple logistic regression showed the following factors to be independently related to the development of surgical complications: adenomyosis, surgical time, bowel resection and hysterectomy. The attributed risk of each factor is shown in Table 4. The risk of presenting complications increased 4.56 times in the $\mathrm{A}$ group ( $\mathrm{Cl}: 1.9-11.3 ; \mathrm{p}=0.001)$. Moreover, there was a statistically significant association between the number of criteria of adenomyosis and the proportion of patients presenting a surgical complication $(p<$ 0.001) (Fig. 1).

Table 4

Risk factors related to surgical complications: multivariate logistic regression analysis

\begin{tabular}{|llll|}
\hline & Odds Ratio & $95 \% \mathrm{Cl}$ & P Value \\
\hline Adenomyosis & 4.558 & $1.845-11.26$ & .001 \\
\hline Surgical time & 1.010 & $1.004-1.016$ & .002 \\
\hline Bowel resection & 2.558 & $0.843-7.761$ & .097 \\
\hline Hysterectomy & 3.110 & $1.293-7.478$ & .011 \\
\hline Cl: confidential interval & & \\
\hline
\end{tabular}

A higher $\mathrm{CD}$ complication rate was also observed in the A group when excluding patients with hysterectomy in both groups (Table 5). 
Table 5

Surgical data considering patients without hysterectomy

\begin{tabular}{|c|c|c|c|}
\hline & $\begin{array}{l}\text { ADENOMYOSIS }(\mathrm{A}) \\
\mathrm{N}=39\end{array}$ & $\begin{array}{l}\text { NO ADENOMYOSIS } \\
\text { (nOA) N = } 62\end{array}$ & $\begin{array}{l}\mathrm{P} \\
\text { Value }\end{array}$ \\
\hline Surgical time minutes (mean $\pm S D$ ) & $226.1 \pm 105.7$ & $185.52 \pm 81.35$ & .032 \\
\hline $\begin{array}{l}\text { Difference pre-post Hemoglobin levels } \\
\mathrm{g} / \mathrm{dl}(\text { mean } \pm \mathrm{SD})\end{array}$ & $2.22 \pm 1.41$ & $1.85 \pm 1.11$ & .175 \\
\hline Hospital Stay days (mean \pm SD) & $3.85 \pm 4.59$ & $2.58 \pm 1.76$ & .02 \\
\hline CD Complications & $13(33.33)$ & $5(8)$ & .003 \\
\hline$n(\%)$ & $7(17.94)$ & $3(4.83)$ & \\
\hline I & $5(12.82)$ & $2(3.22)$ & \\
\hline II & $1(2.56)$ & $0(0)$ & \\
\hline III & $0(0)$ & $0(0)$ & \\
\hline IV & & & \\
\hline
\end{tabular}

\section{Discussion}

The possibility of identifying predictable preoperative features that could modify surgical results would be of great interest [19]. The results of our study suggest that the presence of adenomyosis increases the surgical complication rate in DE patients. Furthermore, this increased risk is related to the number of TVS adenomyosis criteria.

While the impact of adenomyosis on surgical results has been assessed in other benign conditions, to our knowledge no previous study has evaluated the impact of adenomyosis on surgical complications in women with DE undergoing laparoscopic surgery [20]. Previous studies in benign pathology reported an increased rate of bladder and ureteral complications for vaginal hysterectomy in patients with only adenomyosis [21] but this was not observed with the laparoscopic approach [22]. Accurate preoperative imaging assessment has been described in cases of bowel DE, and several previous studies have reported a high accuracy of up to $89.9 \%$ and $98.1 \%$ in correlating TVS findings with surgical difficulties $[11,16]$. These studies are primarily based on ultrasound findings of ovarian mobility and the presence or not of bowel endometriosis but did not specifically included the presence of adenomyosis.

Due to the surgical complexity of DE procedures, even in expert hands, the rate of complications is still considerable especially when colorectal resection is involved, ranging from $3.4-25 \%[19,23,24]$. In our study, clinically relevant complications (CD type III) were only recorded in $2.54 \%$ of cases. These complications were also related to bowel surgeries, similar to previously published data [13, 23-25]. 
Hysterectomy is considered the surgical treatment of choice for most women with adenomyosis who do not wish to preserve fertility after medical treatment has failed [26]. Concomitant hysterectomy in the A group was performed in $49.35 \%$ vs. $22.5 \%$ in the noA group ( $p$ 0.002). Hysterectomy in the context of DE has been shown to be associated to longer operative time [27]. The application of a multivariate model demonstrated that hysterectomy was an independent factor with or without adenomyosis. This was also confirmed in the subanalysis of surgical outcomes between groups performed, considering only patients without hysterectomy.

In addition, all the factors that may contribute to a higher risk of complications (bowel and urinary surgery, previous endometriosis surgeries and operative time) were adjusted in the multivariate analysis in order to prevent bias.

On the other hand, Van den Bosch et al. [8] proposed a new classification system which includes different adenomyosis subtypes, including intrinsic/extrinsic adenomyosis, adenomyosis externa and focal adenomyosis in the outer myometrium (FAOM) $[3,28]$. Focal adenomyosis commonly affects the external part of the myometrium and may attach the uterus to other structures, hindering surgery and increasing the risk of presenting surgical complications and impairing other surgical outcomes and may be a possible explanation for our study results although a definite reason is unknown. Moreover, the chronic inflammation, proliferation and fibrosis that supports the pathogenesis of adenomyosis [1] may be another feasible explanation, creating an inflammatory environment more likely to present an increased risk of surgical complications. Further prospective studies are needed to support this correlation with the type of adenomyosis (since this classification was published after performing the present study) and to find a pathogenic mechanism explaining the clinical presentation of our surgical results.

This study has several strengths: it is the first to describe the impact of adenomyosis on surgical outcomes by conditioning the presentation of a surgical complication. Second, the TVS evaluation was performed by two expert sonographers who have previously demonstrated a high diagnostic TVS accuracy for determining the presence of DE (sensitivity $100 \%$, specificity $96 \%$ ) [5]. Moreover, all surgeries were performed by the same team of experienced surgeons in advanced laparoscopy surgery focused on endometriosis.

However, some limitations need to be acknowledged. This was a retrospective, single-center study. In the absence of previous studies, no formal power calculation was performed for sample size determination. Secondly, a patient selection bias may be present. The patients were probably more complex since they were referred to the Endometriosis Unit of a tertiary university center and were, therefore, associated with a higher degree of surgical difficulty and may not be comparable with the general population.

\section{Conclusion}

According to our findings, adenomyosis is an independent preoperative risk factor for surgical complications after adjustment for known demographic, clinical and surgical risk factors. Thus, patients with a preoperative diagnosis of DE and adenomyosis programmed to undergo surgery should be 
informed about the possible development of surgical complications and ideally attended in a referral center by an expert multidisciplinary endometriosis team. However, further prospective studies should be carried out in order to confirm these findings taking into account different adenomyosis phenotypes.

\section{References}

1. Vannuccini, S. et al. Pathogenesis of adenomyosis: an update on molecular mechanisms. Reprod Biomed Online [Internet], 35 (5), 592-601 (2017).

2. Maruyama, S., Imanaka, S., Nagayasu, M., Kimura, M. \& Kobayashi, H. Relationship between adenomyosis and endometriosis; Different phenotypes of a single disease? Eur J Obstet Gynecol Reprod Biol, 253, 191-197 (2020).

3. Chapron, C. et al. Relationship between the magnetic resonance imaging appearance of adenomyosis and endometriosis phenotypes. Hum Reprod, 32 (7), 1393-1401 (2017).

4. Lazzeri, L. et al. Preoperative and postoperative clinical and transvaginal ultrasound findings of adenomyosis in patients with deep infiltrating endometriosis. Reprod Sci, 21 (8), 1027-1033 (2014).

5. Ros, C. et al. Bowel Preparation Improves the Accuracy of Transvaginal Ultrasound in the Diagnosis of Rectosigmoid Deep Infiltrating Endometriosis: A Prospective Study. J Minim Invasive Gynecol, 24 (7), 1145-1151 (2017).

6. Guerriero, S. et al. Systematic approach to sonographic evaluation of the pelvis in women with suspected endometriosis, including terms, definitions and measurements: a consensus opinion from the International Deep Endometriosis Analysis (IDEA) group. Ultrasound Obstet Gynecol. 2016 Sep 1;48(3):318-32.

7. Van Den Bosch, T. et al. Terms, definitions and measurements to describe sonographic features of myometrium and uterine masses: A consensus opinion from the Morphological Uterus Sonographic Assessment (MUSA) group. Ultrasound Obstet Gynecol. 2015 Sep 1;46(3):284-98.

8. Van den Bosch, T. et al. Sonographic classification and reporting system for diagnosing adenomyosis. Ultrasound Obstet Gynecol, 53 (5), 576-582 (2019).

9. Chapron, C. et al. Diagnosing adenomyosis: An integrated clinical and imaging approach. Hum Reprod Update, 26 (3), 392-411 (2020).

10. Exacoustos, C. et al. Ultrasound mapping system for the surgical management of deep infiltrating endometriosis. Fertil Steril, 102 (1), 143-1502 (2014).

11. Menakaya, U. et al. Performance of ultrasound-based endometriosis staging system (UBESS) for predicting level of complexity of laparoscopic surgery for endometriosis. Ultrasound Obstet Gynecol, 48 (6), 786-795 (2016).

12. Kho, R. M. et al. Surgical treatment of different types of endometriosis: Comparison of major society guidelines and preferred clinical algorithms. Best Pract Res Clin Obstet Gynaecol, 51, 102-110 (2018). 
13. Singh, S. S., Gude, K., Perdeaux, E., Gattrell, W. T. \& Becker, C. M. Surgical Outcomes in Patients With Endometriosis: A Systematic Review. J Obstet Gynaecol Canada, 42 (7), 881-88811 (2020).

14. Chapron, C., Marcellin, L., Borghese, B. \& Santulli, P. Rethinking mechanisms, diagnosis and management of endometriosis. Nat Rev Endocrinol, 15 (11), 666-682 (2019).

15. Keckstein, J. et al. Recommendations for the surgical treatment of endometriosis. Part 2: deep endometriosis. Hum Reprod Open, 2020 (1), 1-25 (2020).

16. Tompsett, J. et al. Ultrasound-Based Endometriosis Staging System: Validation Study to Predict Complexity of Laparoscopic Surgery. J Minim Invasive Gynecol, 26 (3), 477-483 (2019).

17. Revised American Society for Reproductive Medicine classification of endometriosis: 1996. Fertil Steril. 1997 May;67(5):817 - 21.

18. Dindo, D., Demartines, N. \& Clavien, P. A. Classification of surgical complications: a new proposal with evaluation in a cohort of 6336 patients and results of a survey. Ann Surg, 240 (2), 205-213 (2004 Aug).

19. Abrão, M. S., Andres, M. P., Barbosa, R. N., Bassi, M. A. \& Kho, R. M. Optimizing Perioperative Outcomes with Selective Bowel Resection Following an Algorithm Based on Preoperative Imaging for Bowel Endometriosis. J Minim Invasive Gynecol, 27 (4), 883-891 (2020).

20. Struble, J., Reid, S., Bedaiwy, M. A. \& Adenomyosis A Clinical Review of a Challenging Gynecologic Condition. J Minim Invasive Gynecol [Internet] 2016;23(2):164-85. Available from:

http://dx.doi.org/10.1016/j.jmig.2015.09.018

21. Furuhashi, M., Miyabe, Y., Katsumata, Y., Oda, H. \& Imai, N. Comparison of complications of vaginal hysterectomy in patients with leiomyomas and in patients with adenomyosis. Arch Gynecol Obstet, $262(1-2), 69-73$ (1998).

22. Meikle, S. F., Nugent, E. W. \& Orleans, M. Complications and recovery from laparoscopy-assisted vaginal hysterectomy compared with abdominal and vaginal hysterectomy. Obstet Gynecol, 89 (2), 304-311 (1997 Feb).

23. Kondo, W. et al. Complications after surgery for deeply infiltrating pelvic endometriosis. BJOG An Int J Obstet Gynaecol, 118 (3), 292-298 (2011).

24. Darai, E., Ackerman, G., Bazot, M., Rouzier, R. \& Dubernard, G. Laparoscopic segmental colorectal resection for endometriosis: Limits and complications. Surg Endosc Other Interv Tech, 21 (9), 15721577 (2007).

25. Nicolaus, K. et al. Extensive endometriosis surgery: rASRM and Enzian score independently relate to post-operative complication grade. Arch Gynecol Obstet, 301 (3), 699-706 (2020).

26. Ajao, M. O. et al. Persistence of Symptoms After Total vs Supracervical Hysterectomy in Women with Histopathological Diagnosis of Adenomyosis. J Minim Invasive Gynecol, 26 (5), 891-896 (2019).

27. Uccella, S. et al. Impact of endometriosis on surgical outcomes and complications of total laparoscopic hysterectomy. Arch Gynecol Obstet, 294 (4), 771-778 (2016).

28. Guo, S. W. The Pathogenesis of Adenomyosis vis-à-vis Endometriosis. J Clin Med, 9 (2), 485 (2020). 
29. Drs Meritxell Gracia, Cristian de Guirior, Marta Valdés-Bango, Mariona Rius, Cristina Ros, Isabel Matas, Marta Tortajada, María Ángeles Martínez-Zamora, Lara Quintas and Francisco Carmona have no conflicts of interest or financial ties to disclose.

\section{Figures}

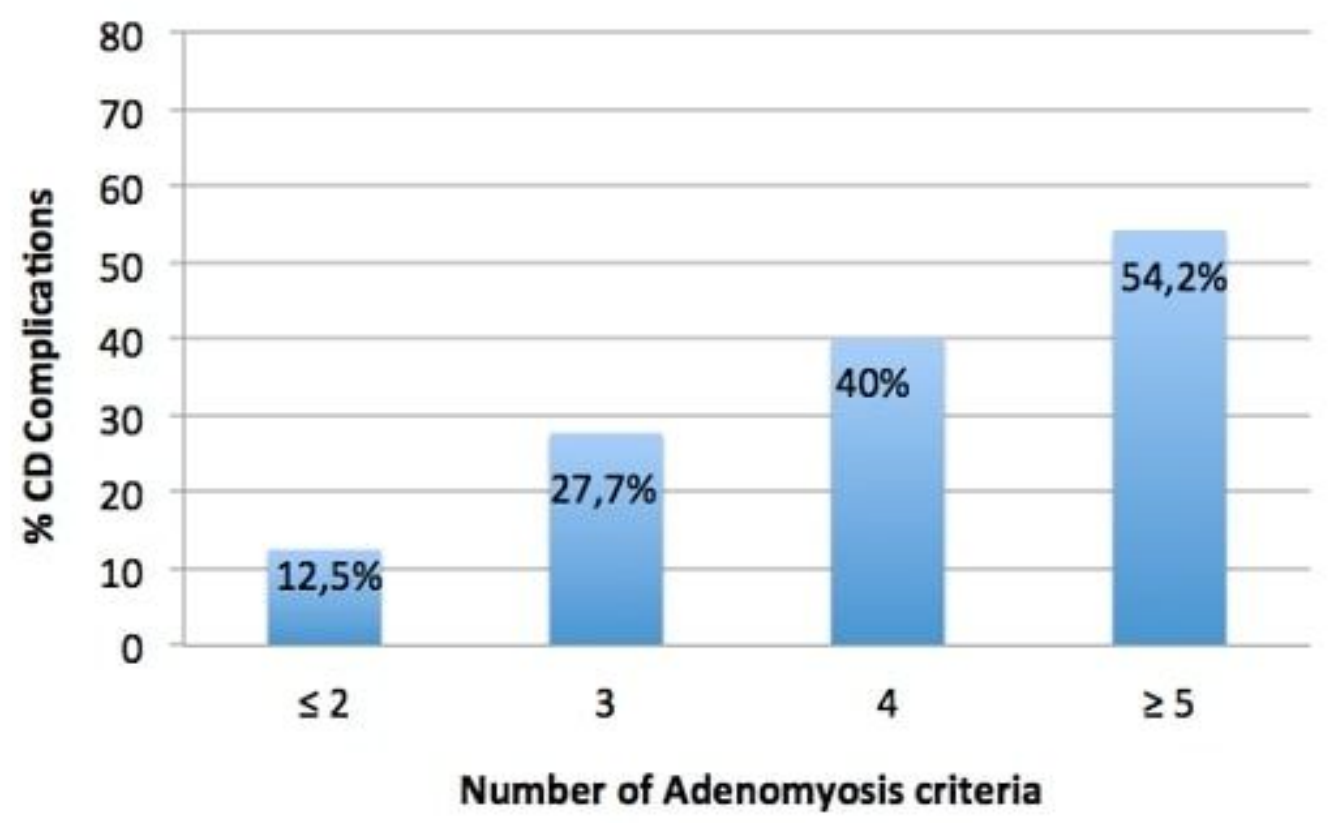

Figure 1

Association between adenomyosis criteria and surgical complications. 